\title{
A Neurocomputational Model Implemented on Humanoid Robot for Learning Action Selection
}

\author{
Emeç Erçelik \\ Electrical and Electronics Faculty \\ Istanbul Teechnical University \\ Istanbul, Turkey 34469 \\ Email: ercelike@itu.edu.tr
}

\author{
Neslihan Serap Şengör \\ Electrical and Electronics Faculty \\ Istanbul Teechnical University \\ Istanbul, Turkey 34469 \\ Email: sengorn@itu.edu.tr
}

\begin{abstract}
Computational modeling of neural circuits enhances our comprehension of brain functions. In addition to the simulation of the models which helps to anticipate cognitive processes, embodiment of these models is essential. Such embodiment would provide the setting to explain neural functioning ongoing in real environments under oncoming sensory information besides giving opportunity of implementation of intelligent systems. Even studies pursued in neuroscience seem far from achieving all these aims in intelligent systems, the pre-results using cognitive models are faster than animal experiments in leading further the understanding of cognitive processes and designing related experiments.
\end{abstract}

In this study, a computational model of basal ganglia, thalamus and cortex for action selection is extended with the point neuron approach to obtain a more realistic method to investigate the model in real time task on humanoid robot platform, Darwin-Op. The spiking neural network model of cortex consists of channels for each action to be elected and plastic all-to-all connections from the sensory stimuli to the basal ganglia structures which are modulated with reward.

In the task, the sensory inputs, namely colors, are presented to the humanoid robot and it is expected that these sensory inputs would be associated with the predefined actions by modulating the connections. Furthermore, the rearrangement of these associations with reward is performed after learning is accomplished. In this way, the embodiment of computational model provided more information on the evolution of connections through reward based learning in the action selection circuit.

\section{INTRODUCTION}

To build models of neurological systems rendering cognitive processes helps our understanding of brain. Though conventional models, as animal models, enlightened many mysteries of brain, recently computational neuroscience begin to contribute more and more to our comprehension of neural mechanisms giving rise to intelligent behavior. One means to benefit more from these computational models is the embodiment of these models as neurorobots. Neurorobots would not only provide platforms for systematically dissecting the neuronal circuits responsible for specific behaviours, simulating the effects of genetic defects, lesions, and loss of cells at different levels of brain organisation and modelling the effects of drugs as stated in [13], but also would enhance technology for intelligent systems. Based on the computational model of Basal ganglia-Thalamus-Cortex (BTC) loop for action selection [4], [2], [3], here the task of associating a sensory stimulus with the desired action is realized on humonoid robot. The association is built due to reward learning and this is accomplished by modulating the connections between structures in BTC loop. The computational model in [2] is expanded by using point neurons for cortex (Ctx), to obtain a more realistic model for building association between sensory stimuli and actions.

The task consists of associating sensory inputs, which are three different colors, presented to the humanoid robot with desired actions. Through this task, the associations are built with reward by modulating the plastic connections in the model and the connections between channels of Ctx which provide the neural computation. Eventhough there are neurocomputational models of action selection realized by point neurons [5], embodiment of learning action selection by a humonoid robot is realized in this work.

In Section II, the extended model of BTC loop and the learning circuit are explained first. Then, how the implementation of the computational model is done described and the real time evolution of task is revealed in Section III. After explanation of model and the environment, the experiments and results are presented to further clarify the real time evolution in Section IV. The paper is concluded with the discussion of results in Section V.

\section{A SPIKING NeTWORK MODEL FOR LEARNING ACTION SELECTION}

The neurocomputational model used here to build association between sensory stimulus and desired action consists of two main parts. Decision on an action is accomplished by action selection part which involves a neurocomputational model of BTC loop. The second part modulates the parameters of the action selection part to provide the learning of the selection of desired actions. With the modulation of parameters using temporal difference learning (TDL) method, this part realizes building up the association between sensory stimulus and desired action.

\section{A. Neurocomputational Model}

Basal ganglia (BG) along with the related parts of Ctx and Thalamus (Thl) take part in decision making tasks [1], [2]. In this study, the BTC model considered in [2] and [3] is expanded using spiking neural network model of Ctx which is given in Figure 1. In the expanded model, sensory information which reaches to Ctx is transferred to BG and Thl through Ctx and prosessed there to decide on an action. 


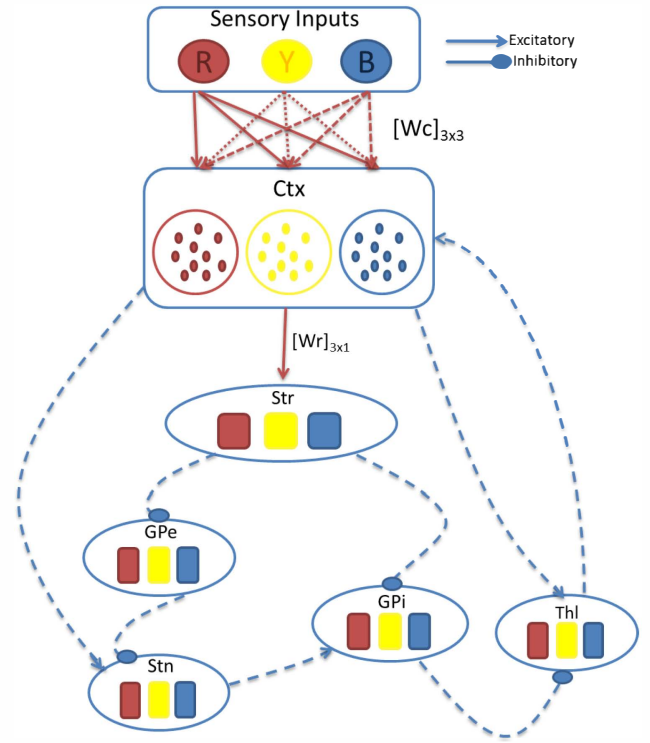

Fig. 1. BTC action selection model. Model is structured with the connections between Ctx, BG substructures and Thl. Ctx part of the model consists of point neurons while the other structures are modeled as mass models. Basal ganglia part consists of Str, GPe, GPi and Stn.

The sensory information transfer is realized by three different pathways: Direct pathway through striatum (Str) and globus pallidus internal $(\mathrm{GPi})$, indirect pathway through Str, globus pallidus external (GPe) and subthalamic nucleus (Stn) and hyperdirect pathway through Stn and GPi [6], [7].

Since there are three sensory information in the considered task, Ctx has three separated neuron populations, which are named channels. In Figure 2, these three neuron populations/channels are indicated by three different colors. In addition, each channel has two neuron groups: excitatory and inhibitory neurons, which are denoted by upper groups and lower groups in Figure 2, respectively. Excitatory neurons make connections only within the channel. They have random connections in themselves and to the inhibitory neurons of the channel. However, inhibitory neurons are connected to the excitatory neurons of each channel. So, when a channel is promoted by a specific sensory stimulus, it inhibites the other channels by its inhibitory neuron group. So neuron populations in Ctx perform winner-take-all competition [8].

Excitatory neurons and inhibitory neurons are modeled as regular spiking and fast spiking point neurons, respectively [9]. There are 80 regular spiking and 20 fast spiking neurons in each channel. All connections are realized with $10 \%$ random connectivity. So, each regular spiking neuron makes eight connections in the excitatory neuron group of the channel and another eight connections to fast spiking neurons of the channel. And each fast spiking neuron makes two random connections to each of three excitatory neuron groups of all channels.

The BG and Thl neurons are modeled as mass models and each of them also has three channels. Ctx, Thl and each element of BG are connected as shown in Figure 1. The excitatory connections are shown as regular arrows and inhibitory connections are shown as point-headed arrows. All the connections are static except the ones between sensory

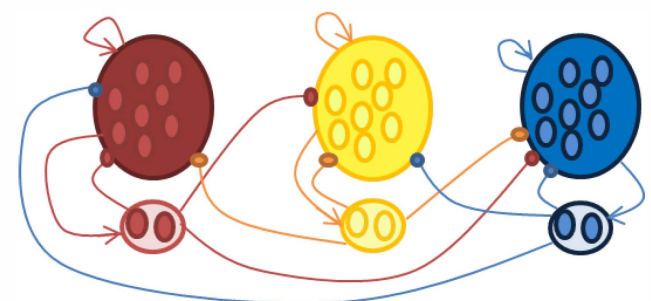

Fig. 2. Spiking neural network model of Ctx. There are three channels in the Ctx model each for a sensory stimulus and each channel consists of 80 regular spiking and 20 fast spiking Izhikevich neurons [9] connectivity of which are $10 \%$. Regular spiking neurons are excitatory (upper neuron groups of each channel) and they have only connections inside its channel. Fast spiking neurons are inhibitory (lower neuron groups of each channel) and they have interchannel connections. So, the connections between channels are provided by inhibitory neurons of each channel.

stimuli and Ctx and between Ctx and Str, which are indicated as $W_{c}$ and $W_{r}$ respectively. These dynamic connections are modified to build up the association between sensory stimuli and actions,

The dynamical system model equations of the BTC model of Figure 1 is given in Equations 1.

$$
\begin{gathered}
\operatorname{Str}(k+1)=W_{r} * f(C t x(k)) \\
\operatorname{GPe}(k+1)=f(-\operatorname{Str}(k)) \\
\operatorname{Stn}(k+1)=f(C t x(k)-G P e(k)) \\
G P i(k+1)=f(\operatorname{Stn}(k)-\operatorname{Str}(k)) \\
\operatorname{Thl}(k+1)=f(\operatorname{Ctx}(k)-G P i(k))
\end{gathered}
$$

Here, $k$ is discrete time variable and all the time dependent neural structure parameters are $3 \times 1$ vectors and their values are in $[0,1]$ range which is provided with tangent hyperbolic function $f$ given in Equation 2 .

$$
f(x)=0.5(\tanh (3(x-0.45))+1)
$$

Ctx values in equations are the scaled mean firing rates of each excitatory group. In this way, the connections and neural structures shown in Figure 1 compose a dynamical system model of BTC circuit.

Now, dynamical connections between sensory stimuli and Ctx, i.e., $W_{c}$ and between Ctx and Str, i.e., $W_{r}$ will be clarified more. Each sensory stimulus, which correspond to red, yellow and blue colors are denoted by R, Y and B letters in Figure 1 , has connections to excitatory neurons of all three channels. Each sensory stimulus connects to all excitatory neurons of the three different channels similarly. The value of promoted input, the input of presented color, is 0.9 while the values of other inputs are 0.1 . In this way, all inputs take part in the learning process. In addition to the sensory stimuli, the excitatory neurons in Ctx have noisy inputs with poisson of $45 \mathrm{~Hz}$. So, there are nine dynamic connections from sensory stimuli to the three different channels of Ctx, which builds up $3 \times 3$ matrix $W_{c}$. Due to this connection structure, before association is built, the sensory stimuli are homogoneously connected to each channel though there are different channels denoting three different colors. 
The other dynamic connection is between Ctx and Str. Each channel of Ctx projects onto the same channel of Str. The projection to $\mathrm{Str}$ is proportional to firing rates of the excitatory neurons of channels in Ctx. Therefore, there are three connections through the channels of Ctx and Str and these connections are indicated as $W_{r}$ which is denoted by a 3x1 matrix. These dynamic connections between sensory stimuli and Ctx and between Ctx and Str are modulated with expectation error of TDL explained in the following subsection.

\section{B. Modulation of Connections}

Modulation of the twelve connections between inputs (I), Ctx and Str are provided according to the TDL. TDL is a method of reinforcement learning [10] that is claimed to be related to reward based learning in BG [11]. TDL modulates the connections by evaluating the expectation error between the expectation of reward as the result of the action chosen with respect to the given sensory inputs and the actual reward that is determined by the environment. The modulation process is provided by the Equations 3 to 7 .

$$
\begin{gathered}
V(k)=W_{v}^{T}(k) I(k) \\
\delta_{c}(k+1)=r+\mu V(k-1)-V(k) \\
W_{v}(k+1)=W_{v}(k)+\eta \delta_{c}(k) I(k) \\
W_{c}(k+1)=W_{c}(k)+\eta \delta_{c}(k) C t x(k) \cdot * I(k) \\
W_{r}(k+1)=W_{r}(k)+\eta \delta_{c}(k) C t x(k) \cdot * \operatorname{Str}(k)
\end{gathered}
$$

In these equations, $k$ is a parameter indicating discrete time. All $k$ dependent parameters are in vector or matrix form except $V$ and $\delta_{c}$ which are scalar variables. $\eta$ and $\mu$ are constants values of which are 0.9. $r$ stands for the reward information coming from environment. Its value is 1 when there is reward and 0 otherwise. In equation $3, V$ indicates the value assigned to the given inputs. This value information is kept in $W_{v}$ which has one weight value for each input, so it is a matrix of dimension $3 \times 1$.

The expectation error is calculated according to the given reward and the difference between previous and current values that is denoted in Equation 4. This expectation error modulates the value weights of inputs $W_{v}$ according to Equation 5 . So, the weights of values are modulated using the input information and the expectation error when there exists sensory information. Also, $W_{c}$ matrix and $W_{r}$ vector, which indicate the weights of connections on the action selection model, are modulated using the expectation error due to the reward obtained as a result of action as seen in Equations 6 and 7.

Thus, whenever there is a difference between the expectation and the actual result the connections between the neural structures, $W_{c}$ and $W_{r}$, are updated proportional to the relation between Ctx and inputs for $W_{c}$ and between Ctx and Str for $W_{r}$. $W_{r}$ determines the projection of information to the BG.

After $\mathrm{Ctx}$ begins to select the desired actions in sequence, the $W_{r}$ connections increase with respect to the expectation error and this increases the projection of information to the BG which effects the learning in long term. In this study, $W_{r}$ connections have a base value that loosely corresponds to the base level of dopamine in the model.

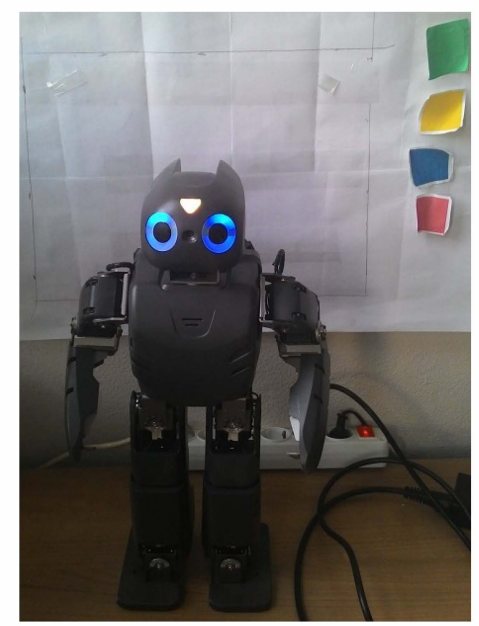

Fig. 3. The robot used in the study is a humanoid robot platform called Darwin-Op. The humanoid robot is expected to associate the presented colors to the desired predefined actions. The three colors to be associated to the actions are red, yellow and blue. The green color is used to indicate reward given when the action choice is the desired one.

\section{IMPLEMENTATION ON HUMANOID ROBOT}

The computational model described in Section II is implemented on a humanoid robot and building the association between sensory stimulus and the desired action is realized. By implementing such a model, compatibility of spike based neural network on a real time task is investigated and a cognitive process is realized. So a step toward realizing a neurorobot which is capable of realizing intelligent behavior with a dynamic model of neural structures is taken.

In the considered task, the robot is expected to associate the presented colors to the desired predefined actions. As shown in Figure 3 on the right side, there are three colors that are yellow, blue and red to be associated to the predefined actions which are head movement, leaning and hand movement, respectively. The green color is used to indicate reward given to the robot if its decision is the desired action corresponding to the color shown. In addition to this, robot is also expected to rearrange the previously associated sensory input-action pairs in the case of no reward. In this way, robot can manage to associate the sensory stimulus to a new desired action by reward and change its previous behaviour. So the implementation of the computational model on humonoid robot also shows the adapdation capacity of model to the changing environment.

The robot used in the study is a humanoid robot platform called as Darwin-Op which is shown in Figure 3. It has $1.6 \mathrm{GHz}$ Atom CPU and $1 G B$ RAM inside and all the calculations are realized on the robot and in real-time. Model is coded in two parts on the humanoid robot which are the simulation of action selection model and learning adaptation to the environment. The action selection model is coded in Python module using NEST simulator for the spiking neural network part [12]. In addition to this, getting sensory input and actuation part is coded in $\mathrm{C}++$ with learning included. The communication scheme of the communicaton between two module can be seen in Figure 4. At first, the humanoid robot gets sensory inputs with its camera and sends this information with the weights of connections, $W_{c}$ and $W_{r}$, to the simulator 


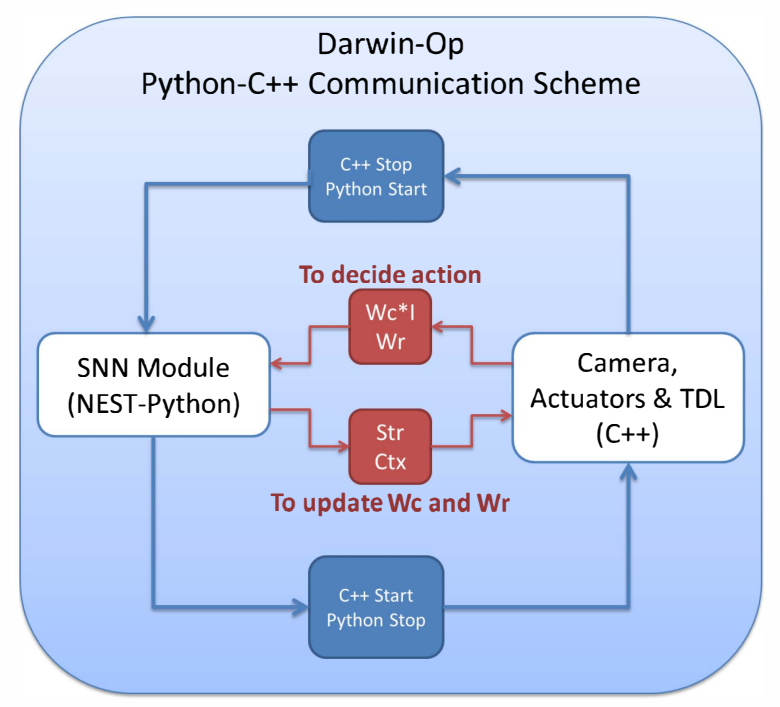

Fig. 4. Model is coded in two parts on the humanoid robot which are the simulation of action selection model and learning together with the communication to the environment. The action selection model is coded in Python using NEST simulator for the spiking neural network part [12]. Getting sensory input and actuation part is coded in $\mathrm{C}++$ including learning. This diagram shows the communication scheme of the communicaton between two modules.

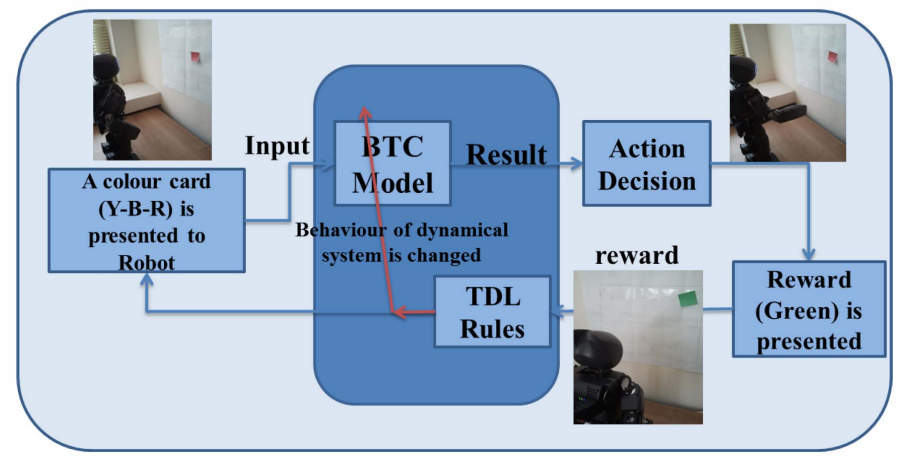

Fig. 5. This diagram shows the evolution of the task. During task, robot tries to learn to associate sensory stimuli to the desired actions. The color information is transmitted to the BTC model as a sensory stimulus in order to get decision from Ctx. After robot performs the selected action, the green card is presented as a reward if the selected action is the desired one. Reward causes an expectation error, which is used to update the values of connections between neural structures of action selection model. Updating values of the connections changes the behavior of dynamical system [4] and so the behaviour of the action selection model. Repeating the reinforcement process, robot is reinforced to associate the desired input-action pair by changing the connections.

part. In the simulator part, Python coded part, the decision is calculated using sensory inputs and connection information. Then the calculated Ctx and Str information is carried to the $\mathrm{C}++$ coded part for getting reward and updating the connection. The two modules wait for the results of the other on real-time process of task.

\section{A. Real Time Implementation of Task on Humonoid Robot}

The task is evaluated as denoted in Figure 5. During task, robot tries to learn to associate sensory stimuli to the desired actions. So, it first recognizes the presented color with its embedded camera that is shown in Figure 5 on the leftside of figure. The color information is carried to the BTC model as a sensory stimulus to get the decision related to coming sensory information from Ctx. The selected action is determined from the mean firing rates of the channels of $\mathrm{Ctx}$ and if mean firing rates of all channels are below a certain threshold then the action is selected randomly. By doing this, humanoid robot is urged to try different actions to get a reward. After robot performs the desired action corresponding to the present sensory information, the green card is presented as a reward. Since values of all $W_{c}$ and $W_{r}$ connections are selected randomly, the robot doesn't know the associations at the beginning of the task. Therefore, robot expects nothing at the beginning. Getting reward while expecting nothing causes an expectation error, which is used to update the values of connections between neural structures of action selection model. Updating values of the connections changes the behaviour of dynamical system [4] and so the behaviour of the action selection model. Repeating the reinforcement process, robot is reinforced to associate the desired input-action pair by changing the connections. After robot successfully manages to associate a given sensory input to the desired action, the expectation error decreases to zero since the robot expects getting reward. However, if robot doesn't get reward when it expects to get, this causes a huge expectation error and robot tries to change the selected action when the sensory input is given. By this way, robot rearranges previously associated pairs and associate the sensory stimulus to a new action.

\section{EXPERIMENTS AND RESULTS}

In this study, the humanoid robot is expected to select the desired actions when specific colors are presented. Thus, it is expected to learn to associate the sensory stimulus to an action by evaluating reward and also to rearrange the previously learnt pair for association to a new action. This task is achieved by updating the connections between sensory stimuli, Ctx and Str as explained in Section III.

To show success of the model in real time learning task, two experiments are realized on humanoid robot. In the first experiment, the sensory inputs are associated to the desired actions in sequence and then the previously associated pair is rearranged. In the second experiment, the sensory inputs are presented to the robot in random order and association time and the strength of the connections are investigated.

Results of the first experiment can be seen in Figure 6. The Figure 6-a shows the number of the presented input (first, second and third), which are red, yellow and blue colors respectively, and the channel of the selected action. The sensory input and the selected actions are indicated with red and blue lines, respectively. The Figure 6-b shows the expectation error, green line, and reward, red line. The Figure 6-c shows the average firing rates of the each channel in the Ctx and red, yellow and blue lines indicate the channels respectively. Through the experiment, the spiking neural network is simulated $200 \mathrm{~ms}$ for each sensory input. The average firing rates of Ctx channels are calculated over the spikes in this $200 \mathrm{~ms}$ time interval. The simulated time of the spiking neural network last $15150 \mathrm{~ms}$ for this experiment, but it takes 45 minutes in real time, real time factor is approximately \%0.6. This is due to the processor inside the robot being not suitable for a spiking neural network simulation in real time. 


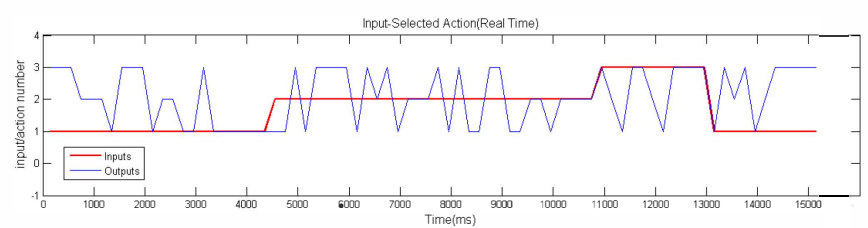

Expectation Error(Real Time)
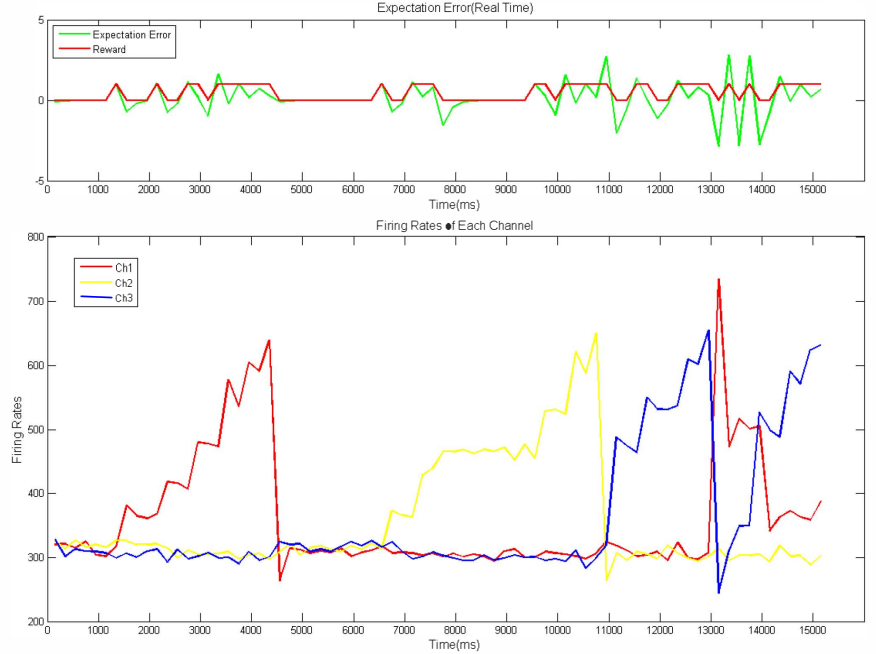

Fig. 6. a (upper figure): The selected actions (blue line) and the sensory inputs (red line). b (middle figure): Reward (red line) and expectation error (green line). c (lower figure): Average firing rates of Ctx channels. The simulated time of the spiking neural network last $15150 \mathrm{~ms}$ for this experiment, but it takes 45 minutes in real time, real time factor (the proportion of simulation time to the real time) of which is approximately \%0.6.

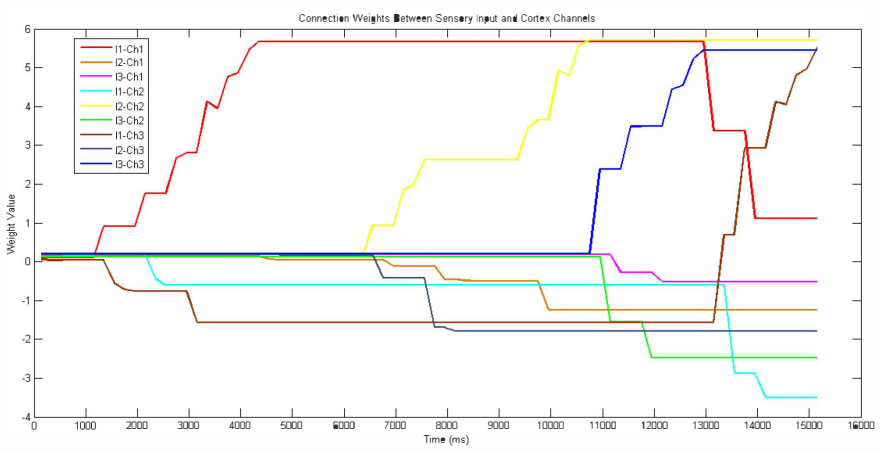

Fig. 7. The evolution of connections between sensory inputs (I) and Ctx channels $(\mathrm{Ch})$ through the first experiment.

During the experiment, the sensory stimuli are first associated with the desired actions which are the first input (red color) to the first action (channel 1), the second input (yellow color) to the second action (channel 2) and third input (blue color) to the third action (channel 3) as seen in Figure 6-a. At the beginning, the humanoid robot selects actions randomly, since there is no winner between Ctx channels until one of the average firing rates reaches to a certain value. After the colors are presented, the expectation error remains zero as far as the first reward to that input is given. This situation can be seen in Figure 6-b,c between the time intervals, $0-1000 \mathrm{~ms}$ and $5000-6500 \mathrm{~ms}$.

When the red color, first input, is presented, the humanoid robot selects a random action until the average firing rate of the first channel reaches to a certain value. Until the first

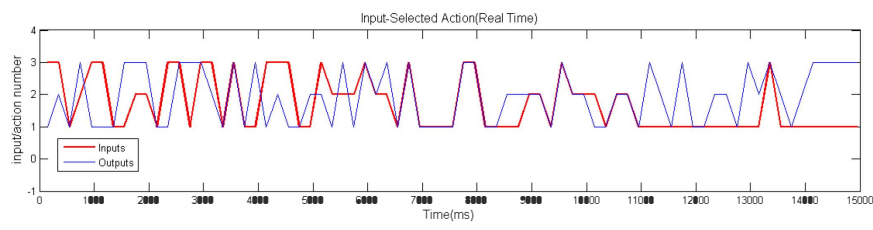

Fig. 8. The selected actions (blue line) and the sensory inputs (red line) of the second experiment. The first input is red color, the second is yellow color and the third is blue color. The sensory stimuli are presented in random order.

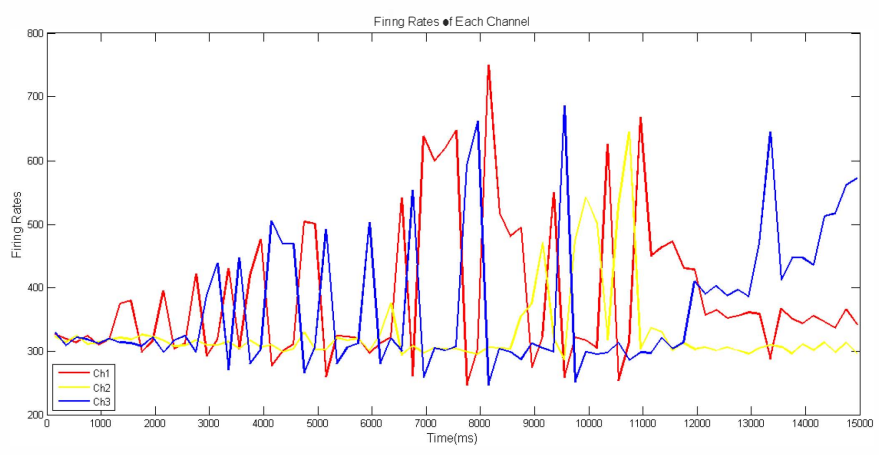

Fig. 9. Average firing rates of Ctx channels through the second experiment.

reward, the expectation error remains zero; this is why the connections and firing rates of channels remains same. This situation can be followed in Figure 6-c between the time intervals, $0-1000 \mathrm{~ms}$ and $5000-6500 \mathrm{~ms}$. In Figure 7 , the evolution of connections between sensory inputs and Ctx channels through the experiment can be followed. At the beginning, the connections have a random value close to zero and evolve to values which build the associations between sensory inputs and desired actions in the way that the expectation error decrease to zero. After all sensory stimuli are associated with the actions, the first sensory stimulus is reassociated to the third action at the end of the experiment to show the realization of rearrangement of associations. After $13000^{t h} \mathrm{~ms}$ the first sensory stimulus is associated to the third action by rewarding selection of the third action instead of the first. Therefore, the connections between the first stimulus and the first action decrease while the connections between the first stimulus and the third action increases (Figure 7). In Figure 6-c, it is seen that the average firing rate of the third channel increases due to the change in the connections. However, the connections between the first input and the first channel is still higher than the value at the beginning. So, they can be reassociated more easily considering the association at the beginning, which is also compatible to the reinforcement learning aspect.

As a second experiment, the sensory inputs are presented in a random order seen in Figure 8. In the second experiment, the first input associated to the first action and so on. After all associations are accomplished the associated action of the first input is changed to be the third action. All processes are the same as the first experiment, but the orders of presented sensory stimulus are random. The average firing rates during the second experiment can be seen in Figure 9. On the $11000^{t h}$ $m s$ all inputs are associated to the desired actions and the rearrangement of association of the first sensory stimulus begins after then. The second experiment is terminated after 15150 


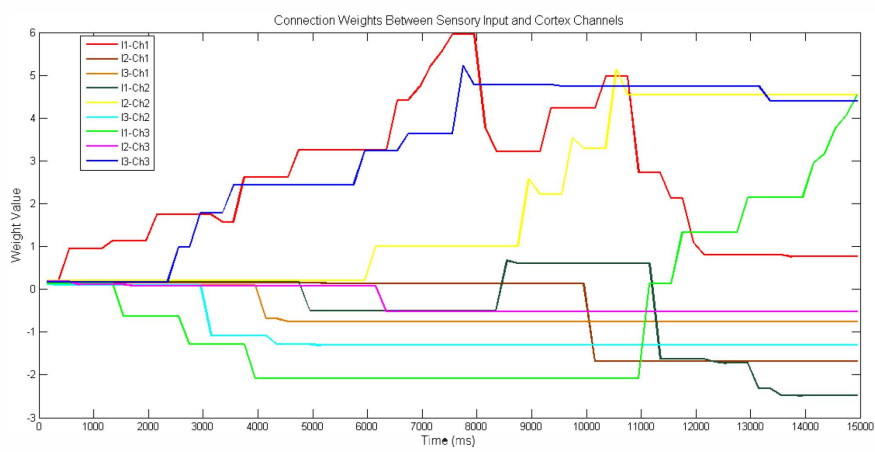

Fig. 10. The evolution of connections between sensory inputs (I) and Ctx channels $(\mathrm{Ch})$ through the second experiment.

$m s$, since the rearrangement is accomplished. In total, the task is accomplished in approximately same time interval in both of the experiments even the learning in the second experiment is realized in random order. The evolution of connections is presented in Figure 10 for the second experiment. Since the actions are selected randomly, when there is no winner, some of the connections are depressed in proportion to the expectation error because of not having expected reward. This situation happens for the connections between I1-Ch3 (green line), I3-Ch2 (cyan line) at the beginning of the experiment. The I1-Ch1 connection (red line) also decreases after $10500^{\text {th }}$ $m s$, but to a value close to one which makes a further association easier.

\section{CONCLUSION}

In this study, learning to build associations between the sensory inputs and actions are realized on a humanoid robot on a real time task. Through the task, an association of the visual sensory inputs to predefined actions are built up. The computational model in [2] is extended for this task with point neurons inside Ctx and reward modulated connections. Since embodiment of the computational models of neuronal circuits is an emerging way of investigating brain organisation, an environment for the realization of action selection circuit and learning is built to simulate the computational model in real time. Neural structures of the BG and the Ctx are modeled simple to decrease the computation need through the task, since the aim is to investigate the applicability of such model on the humanoid robot platform in real time. Despite these kind of humanoid robot platforms have high mobility abilities, they have low computation abilities for embodied simulation of neural circuits. As a result of this, the simulation of 300 point neurons and the dynamical system model has a $0.6-1 \%$ real time factor. One of the important aspect for the embodiment is that since the point neurons in $\mathrm{Ctx}$ are in relation with the BG and Thl structures which are modeled as mass model, the neuron parameters doesn't need to be optimized for a specific task. Therefore, despite the lack of model reality and detail in the computational models of BTC loop for action selection, this simple approach is sufficient from modeling aspect to show the action selection behaviour on $\mathrm{Ctx}$ in real time applications.

As a future research, we aim to increase the real time factor of the model to be used on the humanoid robot platform. This development leads to enlarge the real time task to be more realistic and let the model to be used in search of brain organisation.

\section{ACKNOWLEDGMENT}

The Humanoid Robot Platform is purchased by TUBITAK Project 111E264. We thank to Berat Denizdurduran for his valuable discussions and guidance on expanding and implementing the action selection model.

\section{REFERENCES}

[1] T. J. Prescott, F. M. M. Gonzales, K. Gurney, M. D. Humphries and P. Redgrave, A robot model of the basal ganglia: Behaviour and intrinsic processing, Neural Networks, 19, pp.31-61, 2006.

[2] N. S. Sengor, O. Karabacak and U. Steinmetz, A Computational Model of Cortico-Striato-Thalamic Circuits in Goal-Directed Behaviour, Proceedings of International Conference of Artificial Neural Networks (ICANN'08), Springer-Verlag, pp.328-337, 2008.

[3] B. Denizdurduran and N. S. Sengor, A Realization of Goal-Directed Behavior-Implementing a Robot Model Based on Cortico-StriatoThalamic Circuits. ICAART 2012, Algarve, Portugal, 2012.

[4] B. Denizdurduran, "Learning How To Select An Action: From Bifurcation Theory To The Brain Inspired Computational Model", M.Sc. Thesis, ITU Graduate School of Science Engineering and Technology, 2012.

[5] F. Chersia, M. Mirollia, G. Pezzulob, G. Baldassarrea, "A spiking neuron model of the cortico-basal ganglia circuits for goal-directed and habitual action learning", Neural Networks, 41, pp.212-224, 2013.

[6] G. E. Alexander, M. D. Crutcher and M. R. DeLong, Basal gangliathalamacortical circuits: Parallel substrates for motor, oculomotor, "prefrontal" and "limbic" functions, Progress in Brain Research, 85, pp.119146,1990

[7] M. J. Frank, Hold your horses: A dynamic computational role for the subthalamic nucleus in decision making, Neural Networks, Vol. 19, pp. 1120-1136, 2006.

[8] P. Dominey, M. Arbib, J.P. Joseph, "A Model of Corticostriatal Plasticity for Learning Oculomotor Associations and Sequences", Journal of Cognitiue Neuroscience 7(3) :311-336, 1995.

[9] E. M. Izhikevich, Simple Model of Spiking Neurons, IEEE Transactions on Neural Networks, Vol. 14, No. 6, 2003.

[10] R. S. Sutton, A. G. Barto, Reinforcement Learning: An Introducion. MIT Press, Cambridge, MA, A Bradford Book, 1998.

[11] W. Schultz, P. Dayan, P. Montague, A Neural Substrate of Prediction and Reward, Science, 275, pp.1593-1599, 1997.

[12] M-O. Gewaltig and M. Diesmann, NEST (Neural Simulation Tool), Scholarpedia 2(4):1430, 2007.

[13] H. Markam (Coordinator), "The Human Brain Project", The HBP-PS Consortium, Lausanne, 2012 